\title{
Use of electrospun fiber mats for the remediation of hypersaline geothermal brine
}

\author{
Aslı Çelik ${ }^{\mathrm{a}}$, Gonca Koç ${ }^{\mathrm{b}}$, Emre Erdoğan ${ }^{\mathrm{b}}$, Talal Shahwanc, Alper Baba ${ }^{\mathrm{d}}$, \\ Mustafa M. Demira,* \\ ${ }^{a}$ Department of Material Science and Engineering, Izmir Institute of Technology, Gülbahçe Köyü, Urla, 35430 İzmir, Turkey, \\ Tel.+90 23275075 11; Fax:+90 23275075 29; emails: mdemir@iyte.edu.tr (M.M. Demir), aslicelik@iyte.edu.tr (A. Çelik) \\ ${ }^{b}$ TUZLA Geothermal Energy A.Ş., Alsancak, 35220, İzmir, Turkey, emails: gaksoy@endaenerji.com.tr (G. Koç), \\ emre.erdogan@endaenerji.com.tr (E.Erdoğan) \\ 'Department of Chemistry, Birzeit University, Ramallah, Palestine, email: tshahwan@birzeit.edu \\ ${ }^{d}$ Department of Civil Engineering, Izmir Institute of Technology, Urla, 35430, İmir, Turkey, email: alperbaba@iyte.edu.tr
}

Received 26 March 2016; Accepted 1 August 2016

\section{A B S T R A C T}

Geothermal brines display high contents of various metal ions that can adversely affect surface and groundwater resources. Nevertheless, it is possible to sequestrate these metals and use some of them in certain engineering applications. The aim of this study was to fabricate electrospun chitosan fiber mats and remove heavy metal cations from geothermal brine of the Tuzla geothermal field (TGF) by employing these mats. TGF is located on the Biga Peninsula in the northwestern part of Turkey. The brine of TGF has high salinity $(\mathrm{EC}>91 \mathrm{mS} / \mathrm{cm})$ and high temperature (reservoir temperature is $173^{\circ} \mathrm{C}$ ). The results show that the concentrations of lithium ion in geothermal fluid ranges from 17 to $35 \mathrm{mg} / \mathrm{L}$, with little seasonal variations. A horizontal electrospinning setup was employed to obtain a non-woven mat comprising submicron diameter chitosan fibers at $2.0 \mathrm{kV} \cdot \mathrm{cm}^{-1}$. This material was then utilized as a stable membrane for the removal of metal ions is present in the brine through sorption at $25^{\circ} \mathrm{C}$ overnight. The results showed that the chitosan fiber mats sequestrate various ions in the brine. Under the studied conditions, the material was capable of removing $46 \%, 44 \%, 50 \%, 44 \%, 40 \%, 67 \%$ and $48 \%$ of $\mathrm{Li}^{+}, \mathrm{Mg}^{2+}, \mathrm{Ba}^{2+}, \mathrm{Sr}^{2+}, \mathrm{Mn}^{2+}, \mathrm{Ca}^{2+}$ and $\mathrm{K}^{+}$from the geothermal brine, respectively.

Keywords: Brine; Chitosan; Electrospinning; Geothermal fluid; Lithium

\section{Introduction}

Geothermal brine is a potentially significant source of valuable minerals and metals. It is advantageous to extract metal ions from a geothermal power plant's wastewater. For instance, the Tuzla geothermal field (TGF) located in Çanakkale, Turkey, has $25 \mathrm{mg} / \mathrm{L}$ of $\mathrm{Li}^{+}$and $\sim 140 \mathrm{mg} / \mathrm{L}$ of $\mathrm{Sr}^{2+}$. Recovery of minerals and metals from geothermal brine can be viewed as 'solution mining by nature'.

There are different types of water remediation processes including chemical precipitation, flotation, ion exchange and

* Corresponding author. electrochemical deposition. Adsorption is a well-recognized water treatment method. Attempts to search for low-cost adsorbents that have metal-binding capacities have been frequently reported [1]. The adsorbents may be of mineral, organic or biological origin and may include zeolites, industrial by-products, agricultural wastes, biomass and polymeric materials [2]. Recently, numerous approaches have been studied for the development of cheaper membrane technologies, which are more effective than current technologies in removing cations from geothermal water. Electrospinning provides excellent material properties; in particular, it has a high surface area per unit amount of material, which is important for achieving a high adsorption capacity. 
Electrostatic spinning was first identified when aerosols were applied with high electric potential to a drop of liquid by Bose in 1745 [3,4]. In 1882, the developments in electrospinning were continued by Lord Rayleigh, who investigated how many charges would be needed to spray a drop of fluid. When the applied potential exceeds the charges required to overcome the surface tension of droplets, a jet of electrospray is generated [3,4]. In the 1990s, the electrospinning process received academic attention by Reneker research group because of the possibility of fabricating electrospun polymeric nanofibers for viscous solutions [3]. The electrospinning process is dependent on many parameters, including the type of polymer and its molecular weight distributions, glass-transition temperature, solubility, viscosity, concentration and surface tension of the solution [5]. This process requires three main components: a high voltage power supply to provide an electrical field; a syringe to keep the polymer solution; and an electrically conductive collector [4]. Submicron electrospinning fibers have the ability of sorbing cations in a synthetic salt solution [6]. The application of chitosan fibers in water purification has two advantages: (1) the fiber diameter and (2) the functional groups of chitosan, which include amine groups at the C-2 position, which can be protonated [7].

Chitosan is a well-known bio-based, semi-synthetic sorbent that is obtained by deacetylation of chitin; chitin is collected from the shells of crabs and shrimp, insects and fungi. Chitin (poly $(\beta-(1 \rightarrow 4)-\mathrm{N}$-acetyl-D-glucosamine) is natural and the second-most abundant polymer in the world. When the degree of deacetylation of chitin becomes $50 \%$, the polymer is known as chitosan. The deacetylation process occurs with the substitution of the acetamido group with an amine group [8]. Although chitin is non-soluble in aqueous media, chitosan becomes soluble in acidic aqueous solutions with an increasing degree of deacetylation. The protonation of the $\mathrm{NH}_{2}$ functional groups on the D-glucosamine repeating units provides chitosan with solvation abilities. The $-\mathrm{NH}_{2}$ groups on chitosan possess a good chelation ability with various metal ions [9]. The efficiency of complexing with metal ions can be improved by increasing the degree of deacetylation. Chitosan is a pseudo-natural cationic polymer and can be applied as a solution, film or fiber [10]. Chitosan polymer mixtures can be converted into a nanofibrous mat by an electrospinning process.

In this work, chitosan fibers with submicron diameters have been fabricated at laboratory conditions. The material was subsequently employed in the separation of metal ions from geothermal brine.

\section{Study area}

Tuzla is an active geothermal area located in the northwestern region of Turkey, $80 \mathrm{~km}$ to the south of the city of Çanakkale and $5 \mathrm{~km}$ away from the Aegean Coast (Fig. 1). $\mathrm{NaCl}$-type geothermal brine that is derived from this area has a typical water temperature of $173^{\circ} \mathrm{C}$ [11]. Studies on hydrogeochemistry indicate that geothermal fluids comprise a high concentration of elements including $\mathrm{Li}^{+}$and $\mathrm{Sr}^{2+}$ in the surface, groundwater and soil in the TGF. Geothermal brine is found in shallow volcanic reservoirs at depths between 330 and $550 \mathrm{~m}$. TGF is an interesting area in Turkey because of its temperature and dissolved ions. The chloride concentration of Tuzla geothermal brine reaches $36,000 \mathrm{mg} / \mathrm{L}$, which is nearly twice the concentration of seawater and is termed 'brine'. Furthermore, the sodium concentration reaches 19,000 mg/L [11-16].

In geothermal brine samples from Tuzla, the fluid from the wells is acidic due to an excess of free $\mathrm{CO}_{2}$ (free mineral acidity). This may result from the high partial pressure of this gas in the well. The $\mathrm{pH}$ of the brine varies due to the degassing of the $\mathrm{CO}_{2}$. The temperatures of geothermal brine in wells T9 and T16 in Tuzla geothermal site were $149.1^{\circ} \mathrm{C}$ and $150.6^{\circ} \mathrm{C}$, respectively (Table 1 ). The concentration of metal ions in Tuzla geothermal fluid was measured previously from 2012 to 2015, as represented in Table 1.

\section{Method and materials}

Brine samples were collected either by using a separator on the well heads or at very high temperatures using a webre separator [17]. The purpose of a small webre separator is to separate the vapor and water fractions of a two-phase steam/water mixture discharged from a geothermal well [18]. A centrifugal separation takes place in the first cyclone from which dry steam and gas is discharged. Separated water is directed into the second cyclone, which operates in a slightly flooded condition, and is discharged together with any remaining traces of steam and gas [19]. The water samples were collected into 1-L glass bottles for trace metal analyses. When sampling, all brine samples were filtered through $0.45 \mu \mathrm{m}$ membranes and acidified with $1 \%(\mathrm{v} / \mathrm{v})$ $\mathrm{HNO}_{3}$ on-site to prevent the precipitation of metal hydroxides and carbonates. Inductively coupled plasma mass spectrometry (ICP-MS) was employed to determine concentration of metal ions.

Chitosan powder $\left(M_{v}=1096 \mathrm{kDa}, \mathrm{MMW}\right.$ and $70 \%-75 \%$ degree of deacetylation) and 1,1,1,3,3,3-hexafluoroisopropanol (HFIP) were obtained from Sigma-Aldrich and were used AQ1 in the preparation of the sorption membrane [6].

\section{Experimental study}

The fiber mats used in this study were obtained by electrospinning of chitosan. The fabricated fiber mats were then used for the removal of ions in a mixture of two brine solutions, obtained from T9 and T16 wells, and mixed with a 1:1 (v/v) proportion.

The electrospinning process involves the application of a high electrical potential to a polymer solution supplied via a constant flow rate syringe pump. The method is well known as being a versatile and continuous technique that is compatible with synthetic and natural polymers, nanoparticles, metals and ceramics for the fabrication of micro- and nano-scale fibers with high surface area to volume ratio [3]. During the electrospinning process, the polymer solution is fed at a constant rate by a syringe pump from the tip of the needle. When an electrical potential is applied, the droplet of the polymer liquid takes a conical shape, known as a Taylor cone, until the electrical field value reaches a critical point. Beyond the critical point, the jet of liquid polymer elongates to the electrically grounded collector's surface [20-22]. 


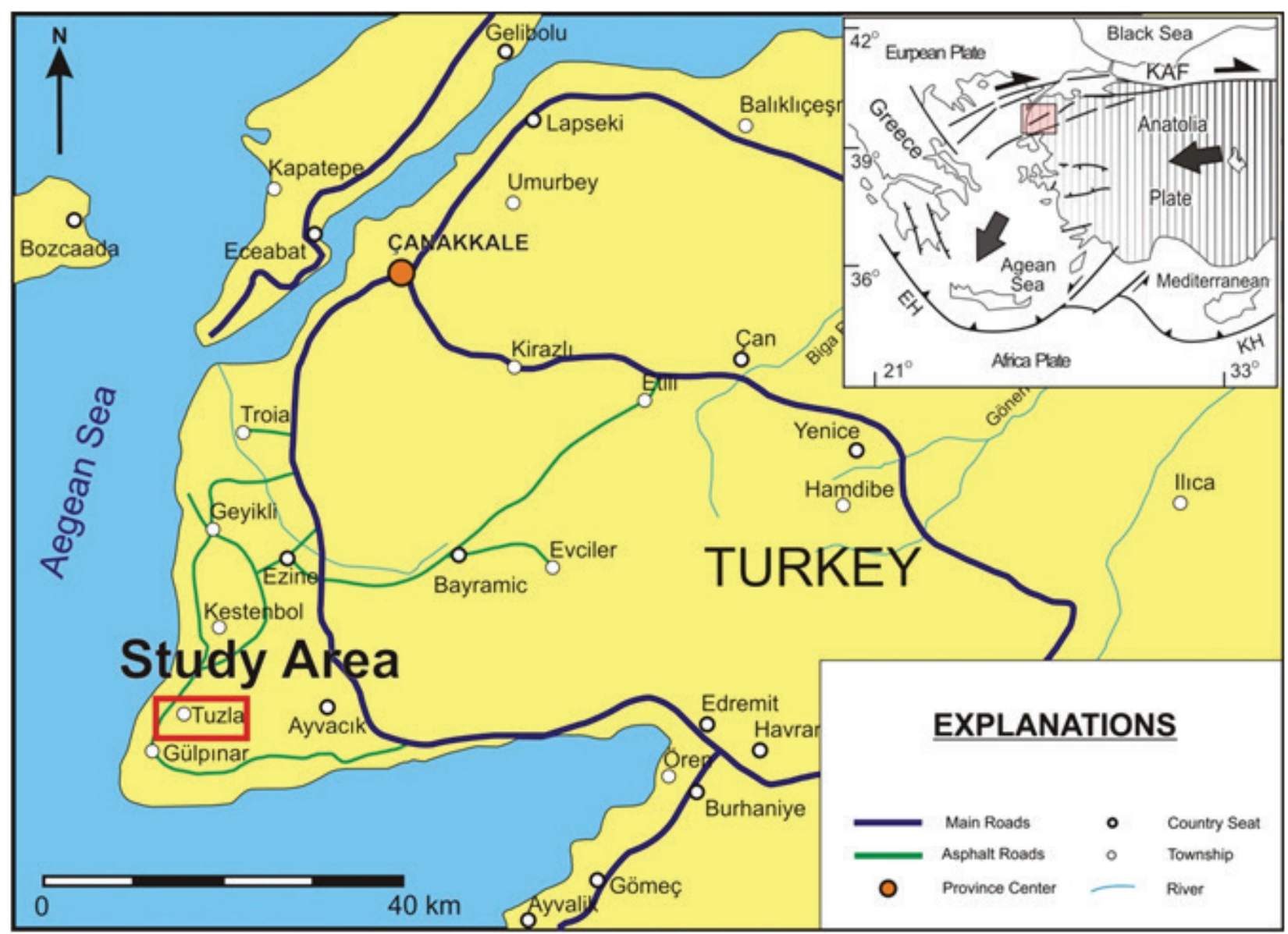

Fig. 1. Location of study area.

Table 1

AQ2 Concentration of metal ions (ppm) in T9 and T16 wells in Tuzla geothermal field

\begin{tabular}{llllllll}
\hline Ions & Sample location & 2012 & October 2013 & April 2013 & September 2014 & July 2015 & March 2016 \\
\hline$\left[\mathrm{Li}^{+}\right]$ & T9 & 21.6 & 33.4 & 26.8 & 19.8 & 17.1 & 18.2 \\
& T16 & 25.6 & 35.0 & 33.1 & 21.1 & 17.0 & 20.2 \\
{$\left[\mathrm{Mg}^{2+}\right]$} & T9 & 82.0 & 138 & 97.0 & 91.0 & 103 & 66.0 \\
& T16 & 109 & 144 & 129 & 103 & 109 & 114 \\
{$\left[\mathrm{Ba}^{2+}\right]$} & T9 & 5.1 & 8.9 & 7.1 & 4.7 & 5.9 & 7.0 \\
& T16 & 5.9 & 9.3 & 8.3 & 4.9 & 5.7 & 6.4 \\
{$\left[\mathrm{Sr}^{2+}\right]$} & T9 & & & 140 & 141 & 141 \\
& T16 & & & 149 & 39 & 141 \\
{$\left[\mathrm{Mn}^{2+}\right]$} & T9 & & 4.5 & 3.0 & 4.3 & 5.4 \\
& T16 & 5.0 & 5.7 & 6.1 & 2.9 & 3.8 & 4.1 \\
{$\left[\mathrm{Ca}^{2+}\right]$} & T9 & 7.4 & 6.1 & & 2,761 & 2,597 & 2,660 \\
& T16 & & & & 2,941 & 2,567 & 2,780 \\
{$\left[\mathrm{~K}^{+}\right]$} & T9 & & & 2,688 & 2,171 & 2,983 & 1,735 \\
& T16 & 1,611 & 2,280 & 2,053 & 2,306 & 2,958 & 2,100 \\
\hline
\end{tabular}

\subsection{Electrospinning of chitosan}

A sample of 19.2-mg chitosan powder was dissolved in $3.0 \mathrm{~mL}$ of HFIP, keeping the concentration constant at $0.4 \%$ $(\mathrm{w} / \mathrm{w})$ for each solution. The solutions were stirred for a week to become homogeneous and clear. The chitosan solutions were subjected to electrospinning. A high voltage generator electrospinning setup was used that comprised a high voltage DC potential supply (Gamma High Voltage Research, 


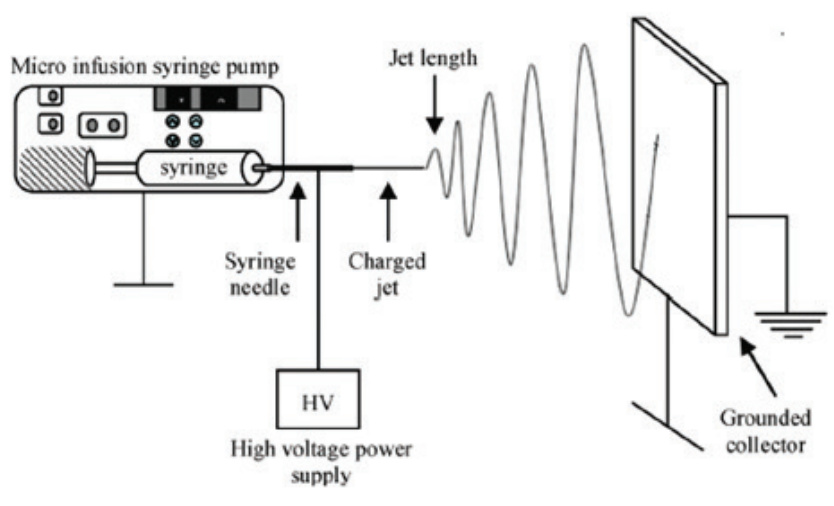

Fig. 2. A schematic illustration of the electrospinning process.

Ormond Beach, FL, USA), a microsyringe pump (LION WZ-50C6) and an aluminum foil as the target media (Fig. 2). The applied potential was $2.0 \mathrm{kV} \cdot \mathrm{cm}^{-1}$ (applied voltage of $14.5 \mathrm{kV}$ ) from the tip of the syringe to the aluminum foil, and the distance was adjusted to $7.0 \mathrm{~cm}$. The polymer solution was immersed at a rate of $4.0 \mathrm{~mL} \cdot \mathrm{h}^{-1}[6,23]$.

\subsection{Sorption studies}

The chitosan electrospun mat was treated with the brine mixture to test its sorption capacity. Two different samples (0.7 and $0.9 \mathrm{mg}$ ) of chitosan electrospun mats were mixed separately with $3.0 \mathrm{~mL}$ of brine fluid mixtures. Before the treatment, the $\mathrm{pH}$ of the geothermal brine was adjusted to 6.0 using a $0.01-\mathrm{M} \mathrm{HNO}_{3}$ solution. The mixture and mats were placed into $15.0-\mathrm{mL}$ centrifuge tubes and continuously shaken in a water shaker (GFL 1083) overnight at room temperature. After treating the electrospun mats with the brine fluid, the mats were separated with a tweezer from the brine mixture and dried in a drying oven at $40^{\circ} \mathrm{C}$. Then chitosan fiber mats were subjected to characterization [6]. The ion concentrations of the brine samples were analyzed before and after treatment with chitosan electrospun mats.

\subsection{Characterization and tools}

The brine was analyzed by ICP-MS [24,25]. This technique is a standard and rapid method for analyzing metal anions or cations. Dilution was applied to both the brine (blank) and chitosan electrospun mat treated brine samples by a factor of 1/40.000 and 1/100.000 (by volume). An ICP-MS (Agilent 7500ce Series) instrument analyzed $\mathrm{Li}^{+}, \mathrm{Mg}^{2+}, \mathrm{Ba}^{2+}, \mathrm{Sr}^{2+}, \mathrm{Mn}^{2+}$, $\mathrm{Ca}^{2+}$ and $\mathrm{K}^{+}$ions in this section. The morphological properties of the chitosan electrospun mat were characterized with an SEM (FEI Quanta 250 FEG) instrument and EDX detector

AQ4 (Oxford Aztek). The chitosan membranes were coated with gold under vacuum.

The chitosan electrospun mats were also scanned with an attenuated total reflectance tool (zinc selenide crystal) at a wave number range of $4,000-1,000 \mathrm{~cm}^{-1}$ in the absorption mode (Fourier transform infrared [FTIR] spectroscopy, PerkinElmer Spectrum 100).

\section{Results and discussion}

The preparation of chitosan fiber is given in Scheme 1. Chitosan fibers were examined by SEM/EDX before (Fig. 3(a)) and after (Fig. 3(b)) treatment with the geothermal brine. The fibers appear to have smooth surfaces and are uniform in diameter. They also look free of beads, which are frequently observed in electrospun fibers. They are uniformly positioned onto counter electrodes. The fibers appear to be homogeneous in terms of thickness. Statistical analysis of 50 randomly selected fibers was performed using the ImageJ program to obtain the diameter distribution of the fibers (Fig. 4(a)). The fiber diameters show a narrow distribution, with the mean diameter being uniformly approximately $160 \mathrm{~nm}$. Upon treatment of the fiber mats with the brine solution, the morphology of the fibers remained unchanged, but salt crystals appeared to precipitate on the surface of the fibers with an approximate diameter of $0.7 \pm 0.3 \mu \mathrm{m}$. The diameter distribution of the salt crystals is given in Fig. 4(b). The size of the crystals is important because when the size is comparable with the inter-fiber porosity, the porosity may be blocked by the crystal precipitates. Thus, the efficiency of the membrane is reduced.

Fig. 5 presents the elemental content on the surface of electrospun fibers as determined by EDX. Note that although Li cannot be detected by EDX, the elemental composition (atomic number $>5$ ) of the sorbed species can be readily obtained. According to the results (Fig. 5), the most abundant element on the electrospun mat $(0.9 \mathrm{mg})$ was $\mathrm{Cl}$, and the next

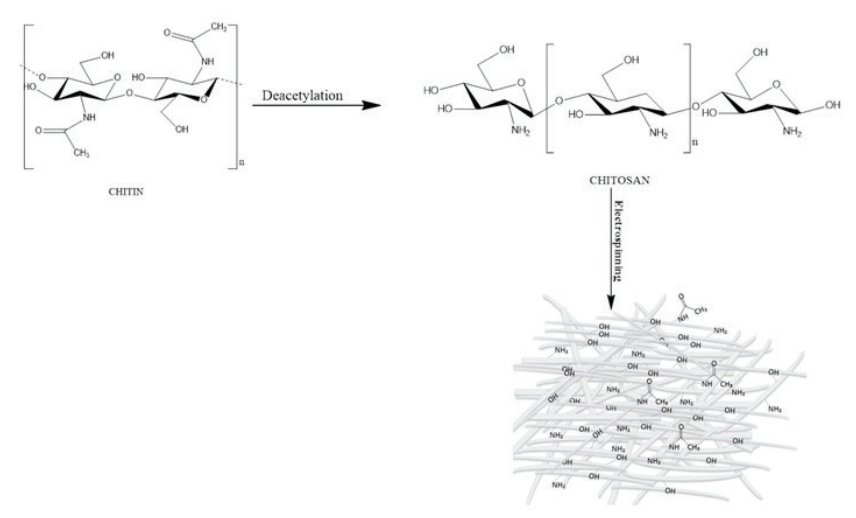

Scheme 1. Cartoon demonstration of the fabrication of chitosan fibers. Note that commercial chitosan was used in this study.
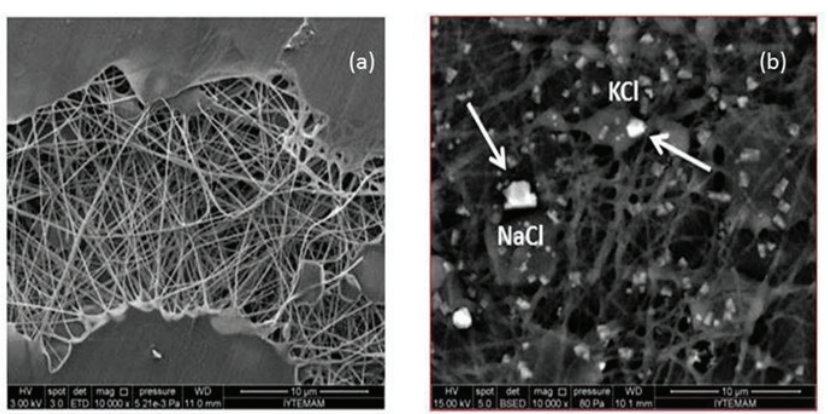

Fig. 3. Typical SEM images of chitosan fiber mat $(0.9 \mathrm{mg})$ before (a) and after (b) treatment with geothermal brine. 

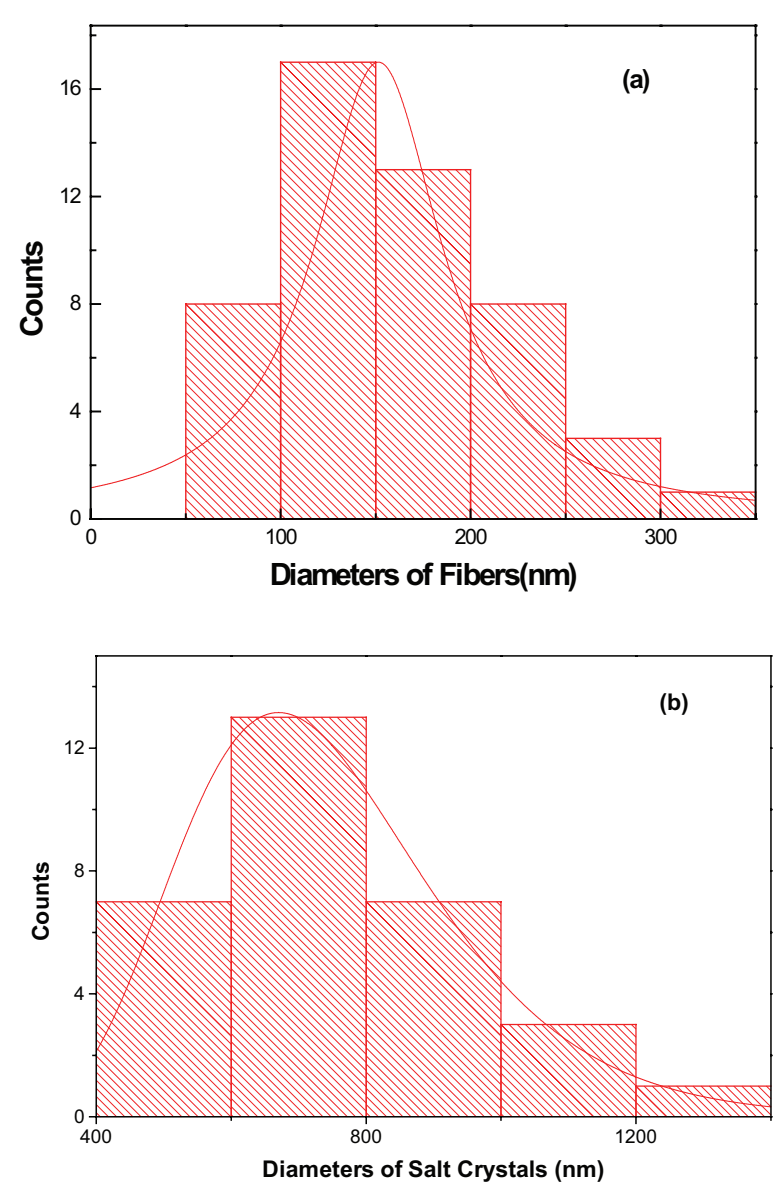

Fig. 4. Fiber (a) and salt crystals (b) diameter distributions of the chitosan fiber mat $(0.9 \mathrm{mg})$.

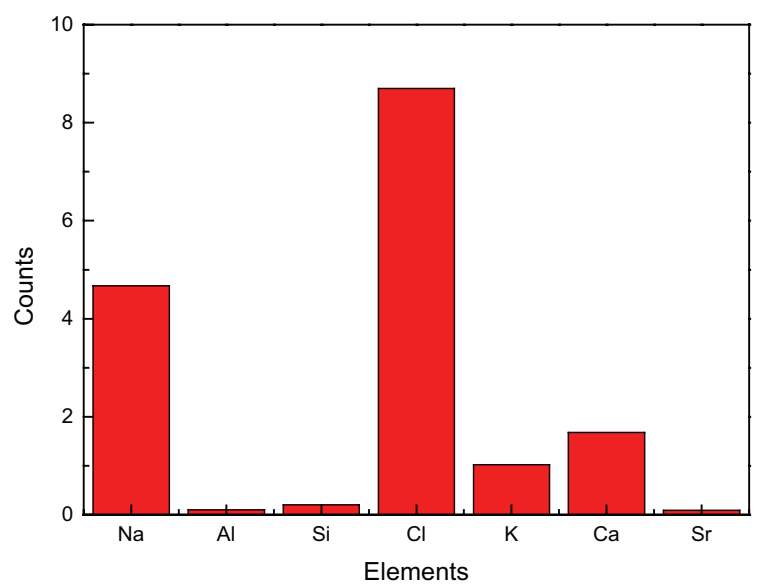

Fig. 5. EDX elemental distribution of various elements on the surface of the chitosan fiber mat $(0.9 \mathrm{mg})$.

one was $\mathrm{Na}$. Considerable amounts of $\mathrm{Ca}$ and $\mathrm{K}$ were also observed. This is in agreement with the SEM images, which showed the formation of both $\mathrm{KCl}$ and $\mathrm{NaCl}$ crystals on the surface of the fibers.

Chemical mapping and EDX studies were performed to reveal the distribution of the most abundant two elements
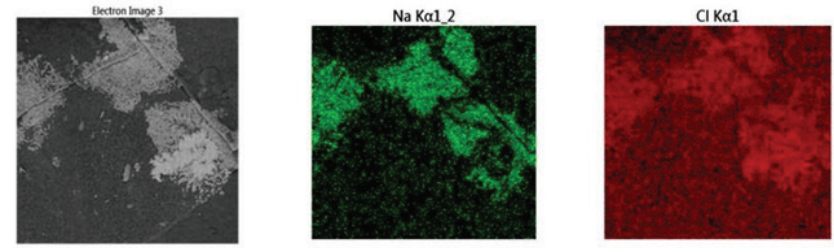

Fig. 6. EDX mapping results $(\times 250 \mu \mathrm{m})$ of the chitosan fiber mat (0.9 mg).

( $\mathrm{Na}$ and $\mathrm{Cl}$ ) on the surface of the fibers. Fig. 6 shows the EDX maps of the electrospun mat and the elemental distributions of $\mathrm{Na}$ and $\mathrm{Cl}$ atoms on its surface. The EDX maps of $\mathrm{Na}$ and $\mathrm{Cl}$ indicated that $\mathrm{Na}$ signals were regionally associated with $\mathrm{Cl}$ signals on the surface of the electrospun mat, possibly originating from $\mathrm{NaCl}$ crystals. Moreover, some of the $\mathrm{Cl}$ signals appeared to cover some other areas in which no sodium signals were observed, presumably due to the formation of $\mathrm{KCl}$.

The extent of removal of various ions by chitosan fiber mats was studied for a given volume of brine mixed with two different quantities of the mats. The initial and final concentrations of various ions were monitored using ICP-MS, and the results are presented in Table 2. According to the ICP-MS analysis results, chitosan fiber mats are capable of holding various ions to an important extent. Increasing the amount of the mats resulted in increased sorbed amounts of ions, as evident from the decrease in the concentration of these ions in the eluent phase. Increasing the quantity of the fiber mats will increase the available surface area of sorption, and the associated important increase in the removal of the ions indicates that the driving force of sorption is far from vanishing; hence, more removal would be expected if more fiber quantities are used.

The data in the table was used to calculate the percent- AQ5 age sorption (or removal) of the ions using the following equation:

$\%$ Sorption $=\frac{[C]_{i}-[C]_{f}}{[C]_{i}} \times 100 \%$

where $[C]_{i}$ is the initial concentration $(\mathrm{mg} / \mathrm{L})$, and $[C]_{f}$ is the final concentration $(\mathrm{mg} / \mathrm{L})$. The obtained results are shown in Fig. 7. According to the figure, while most of the ions demonstrate close sorption percentages, $\mathrm{Ca}^{2+}$ possesses the highest removal percentage. It is important to note that this percentage corresponds to a huge difference in solution concentration of this ion given its large initial concentration. However, it should be mentioned that $\mathrm{Ca}^{2+}$ ions are not expected to be held tightly by the chitosan surface due to the weak chelation ability of these ions with the amine groups of chitosan; hence, sorption is expected to be reversible to an important extent. This might explain the relatively low EDX signals of Ca on dry fiber mats.

It is interesting to note also that, under the employed experimental conditions, about half the concentration of $\mathrm{Li}^{+}$ can be fixed by the fiber mats. This is of particular importance due to the fact that $\mathrm{Li}^{+}$is a rare element with considerable industrial and technological value. The high percentage of $\mathrm{Li}^{+}$removal is in agreement with the high chemical tendency 
Table 2

ICP-MS results of elemental content before and after treatment of brine with chitosan (ppm)

\begin{tabular}{llllllll}
\hline Process & \multicolumn{3}{l}{ Concentrations of ions $(\mathrm{ppm})$} & & & \\
\cline { 2 - 8 } & {$\left[\mathrm{Li}^{+}\right]$} & {$\left[\mathrm{Mg}^{2+}\right]$} & {$\left[\mathrm{Ba}^{2+}\right]$} & {$\left[\mathrm{Sr}^{2+}\right]$} & {$\left[\mathrm{Mn}^{2+}\right]$} & {$\left[\mathrm{Ca}^{2+}\right]$} & {$\left[\mathrm{K}^{+}\right]$} \\
\hline Brine (blank) & 22.0 & 62.0 & 4.0 & 137.0 & 5.0 & $2,384.0$ & $1,615.0$ \\
0.7 mg fiber mat treated brine & 15.0 & 42.0 & 3.0 & 94.0 & 4.0 & 974.0 & $1,244.0$ \\
0.9 mg fiber mat treated brine & 12.0 & 35.0 & 2.0 & 77.0 & 3.0 & 780.0 & 838.0 \\
\hline
\end{tabular}

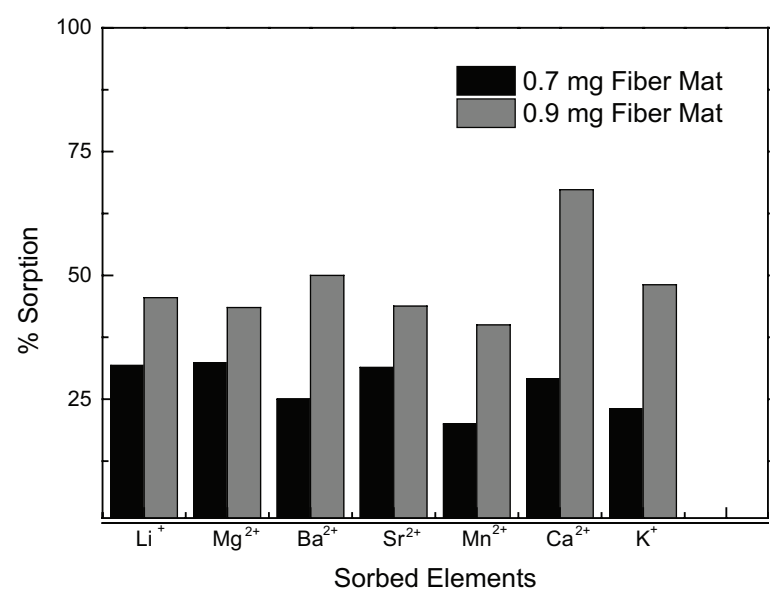

Fig. 7. The percentage sorption of different ions at two mat loadings.

AQ6 of $\mathrm{Li}^{+}$ions to bind to $\mathrm{N}$ in amine groups based on the HSAB principle. As noted earlier, increasing the amount of the fiber mats is expected to lead to higher percentages of $\mathrm{Li}^{+}$fixation.

FTIR is a valuable technique in the investigation of the molecular structure of chitosan and its degree of deacetylation. This technique was used to shed some light on the interaction of the ions in the brine with the chitosan fibers. Fig. 8 shows the spectra of chitosan and brine-treated chitosan. For most of the vibrational signals, both spectra look similar indicating the stability of the material. The spectrum of chitosan shows characteristic absorption bands at 2,878, 1,560, 1,404, $1,320,1,077$ and $1,650 \mathrm{~cm}^{-1}$. These signals can be assigned to the vibrational modes of $-\mathrm{CH}_{3}, \mathrm{~N}-\mathrm{H}$ bending vibrations, $\mathrm{OH}$ (primary alcohol group), $\mathrm{C}-\mathrm{O}-\mathrm{N}$ stretching, $\mathrm{C}-\mathrm{O}$ group and $\mathrm{C}=\mathrm{O}$ stretching, respectively. The broad band in the spectral range $3,000-3,700 \mathrm{~cm}^{-1}$ corresponds to the -OH functional group and to $-\mathrm{NH}$ stretching vibrations in the chitosan structure. Upon treatment with brine, the band broadness decreased, and its intensity increased, possibly reflecting a decrease in the extent of H-bonding in the fiber structure. The changes in this band can also be attributed to interaction of the $-\mathrm{OH}$ and $-\mathrm{NH}$ groups with the cation in the brine solution. Moreover, due to chitosan-ion binding, the $-\mathrm{OH}$ band appears to be shifted from 1,404 to $1,422 \mathrm{~cm}^{-1}$. However, the interaction of $-\mathrm{OH}$ ( or $-\mathrm{NH}$ ) groups with metal ions in brine is expected to be limited since the sorption capacity of chitosan is usually related to the abilities of ions to chelate with the amine groups of chitosan [26]. As can be deduced from the figure, when the fibers are treated with brine, the signals of the amine group at 2,950 and 1,600 $\mathrm{cm}^{-1}$ decrease in their

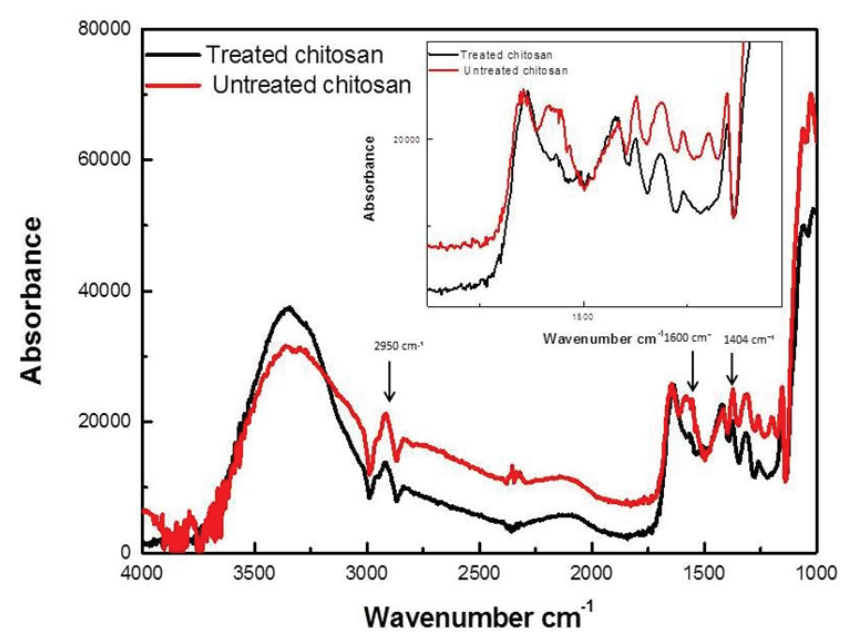

Fig. 8. FTIR analysis of chitosan fiber mat $(0.9 \mathrm{mg})$ before and after treatment with brine.

intensity. Amine groups possess, to varying extents, binding abilities with various cations. The chelation process can yield different forms of coordination and is susceptible to a number of parameters including metal ion selectivity, solution $\mathrm{pH}$, polymer chain length, physical form of chitosan and speciation of metal ions [27]. The reduction in intensity of the amine group may be due to the interaction of this group with metal cations in the brine; however, a more detailed study is required to shed more light on the interaction.

Literature resources contain a large number of studies about various aspects of transition metal sorption on chitosan, including selectivity and mechanisms. However, comparatively less effort has been devoted to characterizing the sorption behavior of chitosan toward group I and II ions, which dominate the brine cationic content. The preliminary results provided in this paper can be further studied; in particular, toward further investigation of the sorption and recovery of $\mathrm{Li}^{+}$ions because of its economic potential.

\section{Conclusion}

Deep geothermal water migrates upward and discharges at springs that become an important source of metal cations around Tuzla and its surroundings. We have developed a membrane system that comprises a surface that is capable of holding metallic ions. It has been demonstrated that the chitosan fiber mats can efficiently adsorb various cations including lithium, calcium and strontium. Moreover, the fibers are stable under hypersaline brine as no fiber breakage or 
morphology change was observed upon treatment. Thus, the fiber mats obtained via electrospinning are promising materials for the removal of metallic ions from geothermal brines. In future work, the surface of chitosan will be modified using chemical groups to develop selectivity toward certain ions like $\mathrm{Li}^{+}$, which has a potential application in Li-ion batteries.

\section{Acknowledgment}

The authors thank The Scientific and Technological Research Council of Turkey (TUBITAK) for the research project encoded with KBAG-114Z940.

\section{References}

[1] F. Fu, Q. Wang, Removal of heavy metal ions from wastewaters: a review, J. Environ. Manage., 92 (2011) 407-418.

[2] T.A. Kurniawan, G.Y. Chan, W.-h. Lo, S. Babel, Comparisons of low-cost adsorbents for treating wastewaters laden with heavy metals, Sci. Total Environ., 366 (2006) 409-426.

[3] J.Doshi, D.H. Reneker, Electrospinning Process and Applications of Electrospun Fibers, Industry Applications Society Annual Meeting, Conference Record, IEEE, 1993, pp. 1698-1703.

[4] A. Greiner, J.H. Wendorff, Electrospinning: a fascinating method for the preparation of ultrathin fibers, Angew. Chem. Int. Ed., 46 (2007) 5670-5703.

[5] M.M. Demir, B. Ozen, S. Özçelik, Formation of pseudoisocyanine J-aggregates in poly(vinyl alcohol) fibers by electrospinning, J. Phys. Chem. B, 113 (2009) 11568-11573.

[6] N. Horzum, E. Boyac1, A.E. Eroglu, T. Shahwan, M.M. Demir Sorption efficiency of chitosan nanofibers toward metal ions at low concentrations, Biomacromolecules, 11 (2010) 3301-3308.

[7] N. Horzum, T. Shahwan, O. Parlak, M.M. Demir, Synthesis of amidoximated polyacrylonitrile fibers and its application for sorption of aqueous uranyl ions under continuous flow, Chem. Eng. J., 213 (2012) 41-49.

[8] S.R. Acharyulu, T. Gomathi, P. Sudha, Physico-chemical characterization of cross linked chitosan-polyacrylonitrile polymer blends, Der Pharmacia Lettre, 5 (2013) 354-363.

[9] E. Guibal, A. Larkin, T. Vincent, J.M. Tobin, Chitosan sorbents for platinum sorption from dilute solutions, Ind. Eng. Chem. Res., 38 (1999) 4011-4022.

[10] M. Rinaudo, Chitin and chitosan: properties and applications, Prog. Polym. Sci., 31 (2006) 603-632.

[11] M.M. Demir, A. Baba, V. Atilla, M. İnanl, Types of the scaling in hyper saline geothermal system in northwest Turkey, Geothermics, 50 (2014) 1-9.

[12] S. Mützenberg, Nature and Origin of the Thermal Springs in the Tuzla Area, Western Anatolia, Turkey, Active Tectonic of Northwestern Anatolia-The Marmara Poly-Project, 1997,
[13] A. Baba, G. Yuce, O. Deniz, D.Y. Ugurluoglu, Hydrochemical and isotopic composition of Tuzla geothermal field (CanakkaleTurkey) and its environmental impacts, Environ. Forensics, 10 (2009) 144-161.

[14] A. Baba, H. Ozcan, Monitoring and Evaluation of the Geothermal Fluid on Soil and Water in the Tuzla Geothermal Field by GIS, Remote Sensing and GIS for Environmental Studies, Vol. 113, 2004, pp. 138-143.

[15] A. Baba, M.M. Demir, G.A. Koç, C. Tuğcu, Hydrogeological properties of hyper-saline geothermal brine and application of inhibiting siliceous scale via $\mathrm{pH}$ modification, Geothermics, 53 (2015) 406-412.

[16] A. Baba, Geothermal Environmental Impact Assessment with Special Reference to the Tuzla, Geothermal Area, Canakkale, Turkey, 2003.

[17] H.G. Sequeira, Hydrogen Sulphide Dispersion Model for the Miravalles Geothermal Field, Costa Rica and Groundwater Flow and Contaminants Transport Models, United Nations University.

[18] S. Arnórsson, J.Ö. Bjarnason, N. Giroud, I. Gunnarsson, A. Stefánsson, Sampling and analysis of geothermal fluids, Geofluids, 6 (2006) 203-216.

[19] R.G. Arones, Comparative Evaluation on the Performance of Top \& Bottom against Side Tapping Sampling Points from TwoPhase Geothermal Pipelines, Proc. World Geothermal Congress 2015, Melbourne, Australia, 2015.

[20] Y. Zhao, L. Wang, H. Yu, C. Li, G. Jing, R. Tong, Y. Chen, Study on the preparation of hyperbranched polyethylene fibers and hyperbranched polyethylene composite fibers via electrospinning, J. Appl. Polym. Sci., 132 (2015).

[21] K. Garg, G.L. Bowlin, Electrospinning jets and nanofibrous structures, Biomicrofluidics, 5 (2011) 013403.

[22] Y. Liu, M. Park, H.K. Shin, B. Pant, S.-J. Park, H.-Y. Kim, Preparation and characterization of chitosan-based nanofibers by ecofriendly electrospinning, Mater. Lett., 132 (2014) 23-26.

[23] K. Desai, K. Kit, Effect of spinning temperature and blend ratios on electrospun chitosan/poly(acrylamide) blends fibers, Polymer, 49 (2008) 4046-4050.

[24] R. Vinodh, R. Padmavathi, D. Sangeetha, Separation of heavy metals from water samples using anion exchange polymers by adsorption process, Desalination, 267 (2011) 267-276.

[25] D. Verma, M.S. Desai, N. Kulkarni, N. Langrana, Characterization of surface charge and mechanical properties of chitosan/alginate based biomaterials, Mater. Sci. Eng., C, 31 (2011) 1741-1747.

[26] R. Navarro, J. Guzmán, I. Saucedo, J. Revilla, E. Guibal, Recovery of metal ions by chitosan: sorption mechanisms and influence of metal speciation, Macromol. Biosci., 3 (2003) 552-561.

[27] C. Gerente, V. Lee, P.L. Cloirec, G. McKay, Application of chitosan for the removal of metals from wastewaters by adsorption-mechanisms and models review, Crit. Rev. Env. Sci. Technol., 37 (2007) 41-127. 


\begin{tabular}{|l|l|}
\hline \multicolumn{2}{|c|}{ Author Query } \\
\hline AQ1 & Please provide location details of “Sigma-Aldrich". \\
\hline AQ2 & In Table 1, month is missing in 2012 column. Please check. \\
\hline AQ3 & Please provide expansion for "SEM" and "EDX”. \\
\hline AQ4 & Please provide location details of “Oxford Aztek". \\
\hline AQ5 & $\begin{array}{l}\text { Please specify which table is referred in the sentence “The data in the table was used to } \\
\text { calculate the percentage sorption.... }\end{array}$ \\
\hline AQ6 & Please provide expansion for "HSAB”. \\
\hline AQ7 & Please provide English translation of journal title of the reference [8], if available. \\
\hline AQ8 & Please provide publisher details for references [12] and [14]. \\
\hline AQ9 & Please provide complete details for references [16] and [17]. \\
\hline AQ10 & Please provide page range for reference [20]. \\
\hline AQ11 & Please provide page range for reference [21] instead of article number, if available. \\
\hline
\end{tabular}

